\title{
Suspected Fetal Growth Restriction at 37 Weeks: A Comparison of Doppler and Placental Pathology
}

\author{
William M. Curtin, ${ }^{1}$ Karmaine A. Millington, ${ }^{2}$ Tochi O. Ibekwe, ${ }^{3}$ and Serdar H. Ural ${ }^{4}$ \\ ${ }^{1}$ Division of Maternal-Fetal Medicine, Department of Obstetrics \& Gynecology \& Pathology and Laboratory Medicine, \\ Pennsylvania State University College of Medicine, Hershey, PA, USA \\ ${ }^{2}$ Department of Pathology \& Laboratory Medicine, Pennsylvania State University College of Medicine, Hershey, PA, USA \\ ${ }^{3}$ Department of Obstetrics \& Gynecology, Pennsylvania State University College of Medicine, Hershey, PA, USA \\ ${ }^{4}$ Division of Maternal-Fetal Medicine, Department of Obstetrics \& Gynecology \& Radiology, \\ Pennsylvania State University College of Medicine, Hershey, PA, USA \\ Correspondence should be addressed to William M. Curtin; wcurtin@pennstatehealth.psu.edu
}

Received 15 January 2017; Revised 4 March 2017; Accepted 12 March 2017; Published 20 March 2017

Academic Editor: Giuseppe Rizzo

Copyright @ 2017 William M. Curtin et al. This is an open access article distributed under the Creative Commons Attribution License, which permits unrestricted use, distribution, and reproduction in any medium, provided the original work is properly cited.

Objective. Our objective was determining if abnormal Doppler evaluation had a higher prevalence of placental pathology compared to normal Doppler in suspected fetal growth restriction (FGR) of cases delivered at 37 weeks. Study Design. This retrospective cohort study of suspected FGR singletons with antenatal Doppler evaluation delivered at 37 weeks had a primary outcome of the prevalence of placental pathology related to FGR. Significance was defined as $p \leq 0.05$. Results. Of 100 pregnancies 46 and 54 were in the abnormal and normal Doppler cohorts, respectively. Placental pathology was more prevalent with any abnormal Doppler, $84.8 \%$ versus $55.6 \%$, odds ratio (OR) $4.46,95 \%$ confidence interval (CI): $1.55,13.22$, and $p=0.002$. Abnormal middle cerebral artery (MCA) Doppler had a higher prevalence: $96.2 \%$ versus 54.8\%, OR 20.7, 95\% CI: 2.54, 447.1, and $p<0.001$. Conclusion. Abnormal Doppler was associated with more placental pathology in comparison to normal Doppler in fetuses with suspected FGR. Abnormal MCA Doppler had the strongest association.

\section{Introduction}

Fetal growth restriction (FGR) is defined in the antenatal period as an estimated fetal weight (EFW) by ultrasound less than the 10th percentile for gestational age in the United States [1]. International consensus definition of FGR is more comprehensive and incorporates other parameters such as abdominal circumference, gestational age of onset, Doppler indices, and growth deceleration before arriving at the diagnosis of FGR [2]. Doppler evaluation of maternal, fetal, and umbilical vessels has been used in the management of suspected FGR to aid in timing of delivery and theoretically could separate the fetus with a placental problem from the constitutionally small normal fetus. Newborns that are less than the 10th percentile for gestational age are classified as small for gestational age (SGA). Methods to determine whether an SGA newborn also has pathologic growth restriction are imperfect. Obvious physical features of FGR in the SGA infant, uncommon with modern obstetric management, include decreased muscle mass and subcutaneous tissue and skin desquamation [3]. Other observations proposed for diagnosing FGR among SGA newborns include low ponderal index [4] and postnatal catch-up growth [5]. Current management in FGR is designed to avoid stillbirth, incidence 1.1-3.6\%, and deliver the most mature baby as possible $[6,7]$. Up to $70 \%$ of fetuses with suspected FGR may be constitutionally small normal infants and may not be at increased risk for stillbirth, and the remainder (after exclusion of birth defects, congenital infections, and chromosomal abnormalities) will have FGR presumably related to a pathologic placental process $[5,8]$.

The use of umbilical artery Doppler in management of suspected FGR is associated with a reduction in perinatal 
deaths [9]. The relationship of umbilical artery Doppler patterns in FGR to placental pathology is more straightforward when the most severe patterns, absent end diastolic velocity (AEDV) or reversed end diastolic velocity (REDV), are present [10]. In these cases, which are usually delivered markedly preterm because of nonreassuring fetal testing, there is loss of arterial vessels within the villi accounting for the abnormal Doppler patterns. In FGR at later gestational ages the villous vascular tree has a larger capacity and abnormal umbilical artery Doppler patterns are less frequent; the placental pathology is more subtle and the lesions can overlap with normal pregnancies [11]. Late-onset FGR pregnancies with uterine artery and middle cerebral artery (MCA) Doppler abnormalities have been associated with placental lesions of underperfusion [12].

We therefore chose to study the correlation of Doppler abnormalities in fetuses with suspected FGR delivered at 37 weeks' gestation at our institution in order to remove the confounding factor that gestational age has on interpretation of placental pathology and the bias toward more severe placental lesions that are seen in FGR fetuses that require preterm delivery. We hypothesized that the group of suspected FGR fetuses with abnormal Doppler would have a higher prevalence of gross and histopathologic abnormalities found in FGR as compared to the group with normal Doppler.

\section{Methods}

This was a retrospective cohort study of singleton fetuses with an ultrasound estimated fetal weight less than the 10th percentile delivered at Penn State Milton S Hershey Medical Center at 37 weeks' gestation during the time period 2011-2013. The study was approved by the Research Subjects Review Board at the Penn State Milton S Hershey Medical Center. Cohorts were divided into normal and abnormal Doppler and compared with respect to both the presence and number of gross and histopathologic findings in the placenta that were plausible in their relation to the FGR. Suspected FGR was a standard indication for submission of the placenta to pathology; thus all placentas from this group were expected to have had a pathologic examination. Pregnancies with uncertain dating, multiple gestations, fetuses with major birth defects, or viral or parasitic infections were excluded. Ascertainment of gestational age followed standard clinical and ultrasound guidelines [13].

The subjects were identified by viewing the electronic birth $\log$ for all deliveries at 37 weeks' gestation with suspected FGR. The ultrasound reports were reviewed for EFW $<$ 10th percentile within three weeks of delivery. EFW and percentile were calculated by software using biometric parameters $[14,15]$. Ultrasound measurements were performed with 2-5 MHZ curvilinear transducers using the iU22 (Philips Medical Systems, Bothell, WA). All sonography was performed by experienced sonographers dedicated to maternalfetal medicine. EFW and head circumference to abdominal circumference ratio (HC/AC) were recorded for analysis.

Doppler measurements were obtained utilizing standard techniques [16-18]. All subjects had umbilical artery Doppler as part of their ultrasound surveillance at diagnosis of suspected FGR and with serial scans up to the time of delivery. MCA and uterine Doppler evaluation had not been utilized at all for the first year of the study period in the evaluation of suspected FGR. After the first year of the study time period, MCA Doppler was incorporated routinely into the evaluation of suspected FGR by 3 of 4 maternal-fetal medicine faculty members and not at all by one faculty member. Only 1 maternal-fetal medicine specialist also utilized uterine artery Doppler in the evaluation of suspected FGR, but only once during the pregnancy. All Doppler studies were reviewed in the GE PACS system (GE Healthcare, Chicago IL) by one maternal-fetal medicine specialist (WMC). The last measurements performed and recorded prior to delivery were used for analysis. The systolic/diastolic ratio, resistance index, pulsatility index (PI), and peak systolic velocity were calculated using the software on the machine. For the MCA Doppler the cerebroplacental pulsatility ratio $(\mathrm{CPR})$ was calculated by dividing the MCA Doppler PI by the umbilical artery Doppler PI. Abnormal umbilical (PI $>95$ th percentile), MCA (PI or $\mathrm{CPR}<5$ th percentile), or uterine artery (PI $>95$ th percentile) Doppler for gestational age was defined by using standard reference charts [16-18]. Subjects who had at least one abnormal Doppler of any type were placed in the abnormal Doppler cohort and subjects who had only normal Doppler were placed in the normal Doppler cohort.

Placentas were examined according to standard protocol [19]. Placental weight and gross characteristics were obtained from the placental pathology report. Placental slides were retrieved from the archive and were reviewed by a single pathologist (KAM) blinded to the Doppler categorization. Placentas that had one or more gross or histopathologic feature that could be considered contributing to FGR were classified as abnormal placentas. Those placentas with no pathologic features were classified as normal. The following gross placental features were considered abnormal: placental weight $<5$ th percentile for gestational age [20], single umbilical artery, marginal or velamentous cord insertion, bilobed or succenturiate placenta, and circummarginate or circumvallate placenta. Additional gross placental findings that were categorized as abnormal included infarcts, abruption, intervillous/subchorionic thrombi encompassing $>5 \%$ of placenta parenchyma, and maternal floor infarction. Histopathologic findings considered contributory to FGR included the following: increased syncytial knots, villous agglutination, increased intervillous fibrin, distal villous hypoplasia, acute atherosis, mural hypertrophy in membrane arterioles, muscularization of basal plate arteries, increased placental site giant cells in decidua basalis, immature intermediate trophoblast in decidua basalis, thin umbilical cord (diameter of the umbilical cord $\leq 8 \mathrm{~mm}$ ), uniformly avascular villi, villous stromal-vascular karyorrhexis, villitis of unknown etiology (VUE) with obliterative fetal vasculopathy, large fetal vessel thrombosis, fetal intimal fibrin cushion, chorangiosis, nucleated red cells in capillaries, and VUE. We followed published guidelines for diagnosis for histopathologic lesions related to FGR [21-23].

Maternal demographic variables collected included age, parity, BMI, race/ethnicity, smoking history, diabetes, hypertension, and mode of delivery. Newborn information collected 
TABLE 1: Abnormal versus normal Doppler antenatal comparisons in suspected FGR.

\begin{tabular}{lccc}
\hline Variable* $^{*}$ & Abnormal Doppler & Normal Doppler & OR (95\% CI) \\
\hline Maternal age & $N=46$ & $N=54$ & 0.044 \\
Maternal BMI & $25.2 \pm 5.7$ & $27.0 \pm 6.2$ & 0.065 \\
Caucasian & $29.6 \pm 6.6$ & $27.4 \pm 4.9$ & 0.158 \\
Diabetes & $28(60.9)$ & $40(74.1)$ & $0.54(0.26,1.48)$ \\
Hypertension & $4(8.7)$ & $3(5.6)$ & $1.62(0.28,9.78)$ \\
Parity $\geq 1$ & $3(6.5)$ & $1(1.9)$ & $3.70(0.34,95.8)$ \\
Smoking & $21(45.7)$ & $31(57.4)$ & $0.62(0.26,1.48)$ \\
EFW ultrasound (grams) & $16(34.8)$ & $16(29.6)$ & $1.27(0.50,3.20)$ \\
EFW $<$ 3rd percentile & $2138.4 \pm 202.0$ & $2167.8 \pm 336.8$ & 0.331 \\
HC/AC $>$ 95th percentile & $13(28.3)$ & $12(22.2)$ & 0.241 \\
Induction of labor & $16(34.8)$ & $20(37.8)$ & 0.582 \\
Vaginal delivery & $38(82.6)$ & $43(79.6)$ & $0.38(0.51,3.76)$ \\
Cesarean for nonreassuring fetal status & $33(71.7)$ & $41(75.9)$ & $0.91(0.37,2.23)$ \\
Vacuum/forceps vaginal delivery & $5(10.9)$ & $1(1.9)$ & 0.487 \\
\hline
\end{tabular}

$\mathrm{OR}=$ odds ratio, $\mathrm{CI}=$ confidence interval, and $\mathrm{FGR}=$ fetal growth restriction.

${ }^{*}$ Results expressed in mean \pm SD or number (\%).

included gender, birthweight, ponderal index/ponderal index $<$ 10th percentile, birthweight percentile [4], birthweight to placental weight ratio [24], NICU admission, days in hospital, hyperbilirubinemia requiring phototherapy, hypoglycemia, hypothermia, and oxygen requirement. SGA was defined as birthweight for the gestational age of 37 weeks of $<2500$ grams [4]. Composite neonatal morbidity was defined as at least one neonatal morbidity, including NICU admission. The data were analyzed by $t$-tests, chi-square tests, and odds ratios with 95\% confidence intervals as appropriate. Statistical analysis was performed using SPSS (Chicago, IL). The primary outcome was the proportion of patients with any placental pathology. Performance characteristics of each Doppler type for identification of placental pathology were calculated. Using data by Dicke et al. [25], in a study of both preterm and term SGA infants that showed 94\% with histopathologic placental lesions in the abnormal Doppler group and $64 \%$ in the normal Doppler group, a sample size of 28 patients in each cohort was calculated to show this difference with a power of $80 \%$ and significance level of 0.05 .

\section{Results}

We identified 177 total patients delivered at 37 weeks' gestation for the indication of suspected FGR; 56 were excluded for EFW > 10th percentile, 8 were with fetal anomalies, and 13 were with multiple gestations, leaving a total of 100 subjects: 54, normal Doppler group and 46, abnormal Doppler group. All 100 subjects were evaluated by umbilical artery Doppler, 68 by MCA Doppler, and 39 by uterine artery Doppler. There were no umbilical artery Doppler patterns of AEDV or REDV. The mean gestational ages of the last Doppler type performed prior to delivery were $36.7 \pm 0.5,36.1 \pm 1.2$, and $32.9 \pm 4.4$ weeks for umbilical, middle cerebral, and uterine artery Doppler, respectively.
Antenatal comparisons of the cohorts abnormal versus normal Doppler are given in Table 1. Maternal age was slightly less in the abnormal group. There were no significant differences in any of the other categories. The newborn comparisons are given in Table 2. Newborns in the abnormal Doppler cohort were significantly lighter and more likely to be SGA. There were no differences in any other comparisons. Overall, 20\% of newborns were admitted to the NICU and $37 \%$ experienced at least one morbidity.

The proportion of placentas with pathologic features compared by Doppler type and cohort is given in Table 3. For any Doppler type utilized, a higher proportion of placental pathology was observed if the Doppler was abnormal, OR = 4.46, 95\% CI: $1.55,13.22$. Of the individual Doppler types, only an abnormal MCA Doppler was significantly associated with placental pathology compared to a normal MCA Doppler, OR $=20.7,95 \%$ CI: 2.54, 447.1.

The performance characteristics for Doppler in the diagnosis of placental pathology are given in Table 4. Doppler had both limited sensitivity and NPV for the detection and exclusion of placental pathology, respectively. All Doppler types performed better on specificity and PPV.

Comparison of the numbers of individual placental abnormalities is given in Table 5. Infarcts were significantly more common in the abnormal Doppler group, $\mathrm{OR}=3.87$, 95\% CI: $1.23,12.67$. Lesions belonging to the category of maternal vascular underperfusion [22] were more common in the abnormal Doppler cohort, OR $=3.75$, 95\% CI: 01.51 , 9.41 .

An analysis of the data comparing groups with $(n=$ 69 ) and without placental abnormalities $(n=31)$ showed birthweights to be lower in the placental abnormality cohort, $2297.7 \pm 234.7$ versus $2452.3 \pm 178.9$ grams, $p=0.002$. There was a higher rate of SGA newborns in the placental abnormality cohort, $42(60.9 \%)$ versus $12(38.7 \%), p=0.040$. There were no differences in newborn morbidities (data not shown). 
TABLE 2: Abnormal versus normal Doppler newborn comparisons in suspected FGR.

\begin{tabular}{lccc}
\hline Variable* $^{*}$ & Abnormal Doppler & Normal Doppler & OR (95\% CI) \\
\hline Birthweight $(\mathrm{g})$ & $N=46$ & $N=54$ & 0.002 \\
SGA & $2268.5 \pm 246.0$ & $2411.2 \pm 193.7$ & 0.004 \\
Ponderal index $\left(\mathrm{g} / \mathrm{cm}^{3}\right)$ & $32(69.6)$ & $22(49.7)$ & $3.33(1.34,8.34)$ \\
Ponderal index $<10$ th percentile & $2.37 \pm 0.33$ & $2.43 \pm 0.33$ & 0.379 \\
Five-minute Apgar $<7$ & $16(34.8)$ & $12(22.2)$ & 0.163 \\
Hospital stay (days) & $1(2.17)$ & $1(1.9)$ & $1.87(0.71,4.96)$ \\
NICU admission & $3(2-16)$ & $3(2-21)$ & $0.35(0.22,0.44)$ \\
Hyperbilirubinemia phototherapy & $9(19.6)$ & $11(20.4)$ & $0.95(0.32,2.82)$ \\
Hypoglycemia & $9(19.6)$ & $9(16.7)$ & $0.95(1.22,3.78)$ \\
Hypothermia & $6(13.0)$ & $3(5.6)$ & $2.55(0.52,13.87)$ \\
Oxygen requirement & $5(10.9)$ & $7(13.0)$ & $0.82(0.21,3.18)$ \\
Composite neonatal morbidity & $4(8.7)$ & $5(9.3)$ & 0.920 \\
Placental weight (g) & $16(34.8)$ & $16(29.6)$ & 0.707 \\
Birth/placental weight ratio & $347.4 \pm 73.4$ & $361.6 \pm 83.0$ & 0.295 \\
Umbilical cord diameter $(\mathrm{cm})$ & $6.72 \pm 1.17$ & $6.94 \pm 1.39$ & 0.748 \\
Placental weight $<$ th percentile & $1.20 \pm 0.35$ & $1.19 \pm 0.24$ & $1.27(0.50,3.20)$ \\
\hline
\end{tabular}

$\mathrm{OR}=$ odds ratio, $\mathrm{CI}=$ confidence interval, and FGR $=$ fetal growth restriction .

${ }^{*}$ Results expressed in mean \pm SD, number (\%), or median (min-max).

TABLE 3: Prevalence of placental pathology: abnormal versus normal Doppler in suspected FGR.

\begin{tabular}{|c|c|c|c|c|c|c|}
\hline \multirow{3}{*}{ Doppler type } & \multirow{2}{*}{\multicolumn{2}{|c|}{$\begin{array}{l}\text { Abnormal Doppler } \\
\text { Placental pathology* }\end{array}$}} & \multicolumn{2}{|c|}{ Normal Doppler } & \multirow{3}{*}{ OR (95\% CI) } & \multirow{3}{*}{$p$ value } \\
\hline & & & \multicolumn{2}{|c|}{ Placental pathology } & & \\
\hline & Yes & No & Yes & No & & \\
\hline Any & $39(84.8)$ & $7(15.2)$ & $30(55.6)$ & $24(44.4)$ & $4.46(1.55,13.22)$ & 0.002 \\
\hline Umbilical & $12(75)$ & $3(25)$ & $57(67.1)$ & $28(32.9)$ & $1.42(0.38,5.81)$ & 0.770 \\
\hline MCA & $25(96.2)$ & $1(3.8)$ & $23(54.8)$ & $19(45.2)$ & $20.7(2.54,447.1)$ & $<0.001$ \\
\hline Uterine & $16(88.9)$ & $2(11.1)$ & $13(61.9)$ & $8(38.1)$ & $4.9(0.74,40.90)$ & 0.074 \\
\hline
\end{tabular}

$\mathrm{OR}=$ odds ratio, $\mathrm{CI}=$ confidence interval, $\mathrm{MCA}=$ middle cerebral artery, and $\mathrm{FGR}=$ fetal growth restriction.

${ }^{*}$ Results expressed in number (\%).

TABLE 4: Performance of Doppler in prediction of placental pathology in suspected FGR.

\begin{tabular}{lcccr}
\hline Doppler & Sensitivity & Specificity & PPV & NPV \\
\hline Any & $55.1(42.6,67.1)$ & $77.4(58.9,90.4)$ & $84.5(70.5,93.5)$ & $43.6(30.3,57.7)$ \\
Umbilical & $17.4(9.3,28.4)$ & $87.1(70.2,96.3)$ & $75.0(47.6,92.7)$ & $32.1(22.4,43.2)$ \\
MCA & $52.1(37.2,66.7)$ & $95.0(75.1,99.9)$ & $96.2(80.4,99.9)$ & $45.2(29.9,61.3)$ \\
Uterine & $55.2(35.7,73.6)$ & $80.0(44.4,97.5)$ & $88.9(65.3,98.6)$ & $38.1(18.1,61.6)$ \\
\hline
\end{tabular}

$\mathrm{PPV}=$ positive predictive value, $\mathrm{NPV}=$ negative predictive value, $\mathrm{MCA}=$ middle cerebral artery, and FGR = fetal growth restriction.

${ }^{*}$ Results expressed in \% (95\% confidence interval).

To study the issue whether the MFM specialists may have been biased in selection of subjects for MCA Doppler, we analyzed cohorts for baseline characteristics and outcomes according to whether or not MCA Doppler was performed and also whether or not uterine artery Doppler was performed. These analyses are given in Tables 6, 7, 8, and 9. We found that those subjects who had MCA Doppler had no difference in their antenatal characteristics in comparison to those who did not with the exception of a lower probability of vaginal delivery, the reason for which is unclear but may be random given the number of variables analyzed. Specific ultrasound parameters, the estimated fetal weight, the proportion with EFW < third percentile, and $\mathrm{HC} / \mathrm{AC}>95$ th percentile did not differ significantly between these two groups. No differences were observed between the groups that had or did not have uterine artery Doppler. We also analyzed the data including only those subjects that had both umbilical and MCA Doppler data, $n=68$, and found no differences in baseline characteristics and outcomes and while the overall OR of any abnormal Doppler having placental pathology increased, the results were not statistically significant from when all subjects $n=100$ were included in the analysis. The results of 
TABLE 5: Occurrence of individual placental abnormality by Doppler cohort in suspected FGR.

\begin{tabular}{|c|c|c|}
\hline & $\begin{array}{c}\text { Abnormal Doppler } \\
\qquad N=46\end{array}$ & $\begin{array}{c}\text { Normal Doppler } \\
\quad N=54\end{array}$ \\
\hline $\begin{array}{l}\text { Placental weight }<5 \text { th } \\
\text { percentile }\end{array}$ & 22 & 18 \\
\hline $\begin{array}{l}\text { Placental configuration } \\
\text { abnormality* }\end{array}$ & 4 & 8 \\
\hline Cord problem ${ }^{* *}$ & 12 & 10 \\
\hline Infarcts & 15 & 6 \\
\hline Abruption & 1 & 0 \\
\hline $\begin{array}{l}\text { Intervillous thrombus }> \\
5 \%\end{array}$ & 1 & 0 \\
\hline $\begin{array}{l}\text { Increased pervillous } \\
\text { fibrin }\end{array}$ & 7 & 4 \\
\hline $\begin{array}{l}\text { Subchorionic thrombus } \\
\text { excessive }\end{array}$ & 2 & 1 \\
\hline $\begin{array}{l}\text { Increased syncytial } \\
\text { knots }\end{array}$ & 1 & 3 \\
\hline Villous agglutination & 3 & 2 \\
\hline Distal villous hypoplasia & 0 & 1 \\
\hline Decidual atherosis & 2 & 0 \\
\hline $\begin{array}{l}\text { Hypertrophy membrane } \\
\text { arterioles }\end{array}$ & 0 & 1 \\
\hline $\begin{array}{l}\text { Muscularization of basal } \\
\text { plate arteries }\end{array}$ & 2 & 0 \\
\hline Avascular terminal villi & 0 & 1 \\
\hline $\begin{array}{l}\text { Large villus intimal } \\
\text { fibrin cushion }\end{array}$ & 0 & 1 \\
\hline Chorangiosis & 1 & 1 \\
\hline VUE & 3 & 0 \\
\hline
\end{tabular}

FGR $=$ fetal growth restriction.

* includes succenturiate lobe, circummarginate, or circumvallate membrane insertion.

** includes thin cord, marginal or velamentous insertion, and single umbilical artery.

these analyses are given in Tables 10, 11, and 12. Logistic regression was performed on the data in Table 10 with variables included in the model: abnormal umbilical artery Doppler, abnormal MCA Doppler, Caucasian ethnicity, maternal age, maternal BMI, EFW on ultrasound, and EFW $<$ third percentile. An abnormal MCA Doppler was the single variable that predicted the presence of placental pathology, adjusted $\mathrm{OR}=45.9,95 \%$ CI: 3.46, 609.6 .

\section{Discussion}

Placental pathology was significantly more common in the group of suspected FGR infants delivered at 37 weeks who had an abnormal Doppler evaluation. There was, however, a high prevalence of placental pathology even in the normal Doppler cohort. This degree of pathology in the group with normal Doppler runs counter to the assumption that the fetus with suspected FGR and normal Doppler is the constitutionally small normal fetus. Our population of FGR fetuses would mainly be considered late-onset FGR, that is, $>32$ weeks [26]. One partial explanation for the high prevalence of placental disease in the normal Doppler cohort would be that even uncomplicated pregnancies have some histopathologic findings. Parra-Saavedra et al. [27] showed that 78\% of late-onset SGA births with normal umbilical artery Doppler had histological placental abnormalities as did $22 \%$ of AGA births. McCowan et al. [28] found that abnormal umbilical artery Doppler reflected earlier and more severe growth restriction in small for gestational age fetuses but was not independently associated with newborn morbidity. They concluded that SGA newborns with normal umbilical artery Doppler were not simply constitutionally small normal infants.

We had few abnormal umbilical Doppler patterns in our study and it is well known that in late-onset FGR umbilical artery resistance is uncommonly elevated in this group and has limited sensitivity in detecting neonatal morbidity [29]. In our study umbilical artery Doppler had the lowest sensitivity of the three Doppler types utilized for the detection of placental pathology. It is unclear why the American College of Obstetricians and Gynecologists only recommends the use of umbilical artery Doppler in the evaluation of suspected FGR [1]. Umbilical artery Doppler, in combination with MCA Doppler, detects centralization of blood flow, also known as "brain-sparing," whereby the fetus increases the blood flow to the brain when there is hypoxia. The MCA Doppler, particularly the CPR, has been shown to have improved sensitivity over umbilical artery Doppler in detection of perinatal morbidity and mortality [29]. In addition, in fetuses with suspected FGR, there is an association between an abnormal MCA Doppler and poorer neurodevelopmental outcomes at 2 years of life [30]. An abnormal MCA Doppler in our study was strongly associated with the presence of placental pathology; these findings are in agreement with those of ParraSaavedra et al. [12].

With respect to newborn outcomes, our study showed the abnormal Doppler group to be of lower birthweight and more likely to be classified as SGA. No difference in neonatal morbidity was noted but our study was not powered to detect differences in secondary outcomes. Overall, $32 \%$ of newborns experienced at least one morbidity and there was a $19 \%$ admission rate to the NICU. This rate of morbidity appears high and brings up questions regarding the ideal gestational age for delivery in late-onset FGR. The Disproportionate Intrauterine Growth Intervention Trial at Term showed lower neonatal intensive care unit admissions after 38 weeks in comparison to 36 to 37 weeks [31].

The strengths of our study were the uniform delivery gestational age of 37 weeks and an institutional guideline that recommends placental examination for all deliveries with suspected FGR. This allowed comparisons of placental pathology not confounded by gestational age or selection bias. The weaknesses were that this was a retrospective study, not all subjects were evaluated by uterine artery and MCA Doppler, and that the Doppler examinations did not occur at the same gestational age. The timing of the uterine artery Doppler evaluations prior to delivery with an average gestational age of 32.9 weeks, considerably shorter than the timing 
TABLE 6: MCA Doppler versus no MCA Doppler antenatal comparisons in suspected FGR.

\begin{tabular}{|c|c|c|c|c|}
\hline Variable ${ }^{*}$ & $\begin{array}{c}\text { MCA, yes } \\
N=68\end{array}$ & $\begin{array}{c}\text { MCA, no } \\
N=32\end{array}$ & OR (95\% CI) & $p$ value \\
\hline Maternal age & $25.9 \pm 6.1$ & $27.9 \pm 5.8$ & & 0.114 \\
\hline Maternal BMI & $28.9 \pm 6.3$ & $27.2 \pm 4.5$ & & 0.119 \\
\hline Caucasian & $46(67.6)$ & $22(68.8)$ & $0.95(0.35,2.56)$ & 0.912 \\
\hline Diabetes & $5(7.4)$ & $2(6.3)$ & $1.19(0.19,9.47)$ & 1.000 \\
\hline Hypertension & $3(4.4)$ & $1(3.1)$ & $1.43(0.12,37.2)$ & 1.000 \\
\hline Parity $\geq 1$ & $36(52.9)$ & $16(50.9)$ & $1.13(0.46,2.83)$ & 0.784 \\
\hline Smoking & $25(36.8)$ & $7(21.9)$ & $2.08(0.72,6.18)$ & 0.138 \\
\hline EFW ultrasound (grams) & $2164.2 \pm 196.5$ & $2133.2 \pm 412.0$ & & 0.689 \\
\hline EFW $<3$ rd percentile & $18(23.5)$ & $9(28.1)$ & $1.27(0.44,3.64)$ & 0.621 \\
\hline $\mathrm{HC} / \mathrm{AC}>95$ th percentile & $23(33.8)$ & $13(40.6)$ & $1.34(0.52,3.46)$ & 0.509 \\
\hline Induction of labor & $54(79.4)$ & $27(84.4)$ & $1.40(0.41,5.01)$ & 0.555 \\
\hline Vaginal delivery & $42(61.8)$ & $27(84.4)$ & $3.34(1.05,11.35)$ & 0.023 \\
\hline
\end{tabular}

$\mathrm{OR}=$ odds ratio, $\mathrm{CI}=$ confidence interval, $\mathrm{MCA}=$ middle cerebral artery, and $\mathrm{FGR}=$ fetal growth restriction .

${ }^{*}$ Results expressed in mean \pm SD or number (\%).

TABLE 7: MCA Doppler versus no MCA Doppler newborn comparisons in suspected FGR.

\begin{tabular}{lccc}
\hline Variable* & MCA, yes & MCA, no & OR (95\% CI $)$ \\
\hline Birthweight $(\mathrm{g})$ & $N=68$ & $N=32$ & 0.752 \\
SGA & $2340.6 \pm 241.6$ & $2356.3 \pm 205.0$ & 0.327 \\
Ponderal index $\left(\mathrm{g} / \mathrm{cm}^{3}\right)$ & $39(57.4)$ & $15(46.9)$ & $1.52(0.60,3.87)$ \\
Ponderal index $<10$ th percentile & $2.40 \pm 0.33$ & $2.41 \pm 0.33$ & 0.862 \\
Five-minute Apgar $<7$ & $20(29.4)$ & $8(25.0)$ & 0.647 \\
Hospital stay (days) & $0(0)$ & $1(3.1)$ & $1.25(0.44,3.63)$ \\
NICU admission & $3(2-16)$ & $3(2-21)$ & $1.53(0.45,5.43)$ \\
Hyperbilirubinemia phototherapy & $15(22.1)$ & $5(15.6)$ & $1.28(0.37,4.61)$ \\
Hypoglycemia & $13(19.1)$ & $5(27.2)$ & $0.94(0.19,5.13)$ \\
Hypothermia & $6(8.8)$ & $3(9.4)$ & $0.82(0.21,3.18)$ \\
Oxygen requirement & $8(11.8)$ & $4(12.5)$ & 0.505 \\
Composite neonatal morbidity & $6(8.8)$ & $3(9.4)$ & $0.93(0.23,4.07)$ \\
Placental weight (g) & $21(30.9)$ & $11(34.4)$ & 0.672 \\
Birth/placental weight ratio & $363.1 \pm 79.7$ & $337.9 \pm 74.6$ & 1.000 \\
Umbilical cord diameter $(\mathrm{cm})$ & $6.64 \pm 1.15$ & $7.26 \pm 1.50$ & 1.000 \\
Placental weight $<$ th percentile & $1.20 \pm 0.30$ & $1.20 \pm 0.30$ & 1.000 \\
\hline
\end{tabular}

$\mathrm{OR}=$ odds ratio, $\mathrm{CI}=$ confidence interval, $\mathrm{MCA}=$ middle cerebral artery, and $\mathrm{FGR}=$ fetal growth restriction.

${ }^{*}$ Results expressed in mean $\pm \mathrm{SD}$, number (\%), or median (min-max).

of the umbilical and MCA Doppler, may have been a factor in its underperformance in prediction of placental pathology.

The impact of the missing MCA Doppler data on the primary outcome variable of prevalence of placental pathology is addressed further. Had this data been present it may have strengthened the association of abnormal Doppler overall with the presence of placental pathology but could have resulted in no change or even weakened the association. We had 32 cases that did not have an MCA Doppler; of these 5 already had an abnormal umbilical artery Doppler and 1 had an abnormal uterine artery Doppler leaving 26 cases of normal umbilical artery Doppler with no MCA Doppler. From our data we know that in this group of subjects about
$1 / 3$ with a normal umbilical artery will have an abnormal MCA Doppler, so this would give an additional 9 subjects in the abnormal Doppler group. The final numbers in the abnormal Doppler group would become $n=55$ and $n=45$ in the normal Doppler group. Assuming all 9 subjects with an abnormal MCA Doppler would have placental pathology, a recalculation of the odds of an abnormal Doppler having placental pathology in comparison to a normal Doppler does not substantially change the results: $48 / 55$ (87.2\%) versus $30 / 45(66.7 \%)$, OR $=3.43,95 \%$ CI: $1.44,10.63$. The data from Table 12, when only the subjects with both umbilical and MCA Doppler $(n=68)$ are analyzed, appear to be in agreement with these calculations. 
TABLE 8: Uterine artery Doppler versus no uterine artery Doppler antenatal comparisons in suspected FGR.

\begin{tabular}{|c|c|c|c|c|}
\hline \multirow{3}{*}{ Variable* $^{*}$} & Uterine artery & Uterine artery & & \multirow{3}{*}{$p$ value } \\
\hline & Yes & No & OR $(95 \% \mathrm{CI})$ & \\
\hline & $N=39$ & $N=61$ & & \\
\hline Maternal age & $25.2 \pm 5.8$ & $27.4 \pm 6.1$ & & 0.079 \\
\hline Maternal BMI & $28.5 \pm 5.6$ & $28.3 \pm 5.0$ & & 0.844 \\
\hline Caucasian & $22(43.6)$ & $22(75.5)$ & $0.42(0.16,1.09)$ & 0.052 \\
\hline Diabetes & $4(10.3)$ & $3(4.9)$ & $2.21(0.39,13.4)$ & 0.427 \\
\hline Hypertension & $2(5.1)$ & $2(3.3)$ & $1.60(0.15,16.8)$ & 0.642 \\
\hline Parity $\geq 1$ & $24(61.5)$ & $28(45.9)$ & $1.89(0.77,4.65)$ & 0.127 \\
\hline Smoking & $16(41.0)$ & $16(26.2)$ & $1.96(0.76,5.04)$ & 0.122 \\
\hline EFW ultrasound (grams) & $2122.0 \pm 203$ & $2174 \pm 322$ & & 0.362 \\
\hline $\mathrm{EFW}<3$ rd percentile & $11(28.2)$ & $14(23.0)$ & $1.32(0.48,3.62)$ & 0.554 \\
\hline $\mathrm{HC} / \mathrm{AC}>$ 95th percentile & $11(28.2)$ & $25(41.0)$ & $0.57(0.22,1.46)$ & 0.194 \\
\hline Induction of labor & $30(76.9)$ & $51(83.6)$ & $0.65(0.21,2.0)$ & 0.406 \\
\hline Vaginal delivery & $24(61.5)$ & $45(73.8)$ & $0.57(0.22,1.47)$ & 0.197 \\
\hline
\end{tabular}

$\mathrm{OR}=$ odds ratio, $\mathrm{CI}=$ confidence interval, and $\mathrm{FGR}=$ fetal growth restriction.

${ }^{*}$ Results expressed in mean \pm SD or number $(\%)$.

TABLE 9: Uterine artery Doppler versus no uterine artery Doppler newborn comparisons in suspected FGR.

\begin{tabular}{|c|c|c|c|c|}
\hline \multirow{3}{*}{ Variable* } & Uterine artery & Uterine artery & \multirow{3}{*}{ OR $(95 \% \mathrm{CI})$} & \multirow{3}{*}{$p$ value } \\
\hline & Yes & No & & \\
\hline & $N=39$ & $N=61$ & & \\
\hline Birthweight (g) & $2338.4 \pm 263.0$ & $2350.3 \pm 207.6$ & & 0.804 \\
\hline SGA & $39(57.4)$ & $15(46.9)$ & $1.52(0.60,3.87)$ & 0.327 \\
\hline Ponderal index $\left(\mathrm{g} / \mathrm{cm}^{3}\right)$ & $2.42 \pm 0.35$ & $2.39 \pm 0.31$ & & 0.658 \\
\hline Ponderal index $<10$ th percentile & $12(30.8)$ & $16(26.2)$ & $1.25(0.47,3.32)$ & 0.622 \\
\hline Five-minute Apgar $<7$ & $0(0)$ & $1(3.1)$ & & 0.320 \\
\hline Hospital stay (days) & $3(2-16)$ & $3(2-21)$ & & 0.496 \\
\hline NICU admission & $9(23.1)$ & $11(18.0)$ & $1.36(0.45,4.08)$ & 0.539 \\
\hline Hyperbilirubinemia phototherapy & $5(12.8)$ & $13(21.3)$ & $0.54(0.15,1.85)$ & 0.281 \\
\hline Hypoglycemia & $3(3.5)$ & $6(9.8)$ & $0.76(0.14,3.76)$ & 1.000 \\
\hline Hypothermia & $3(11.8)$ & $9(14.8)$ & $0.48(0.10,2.14)$ & 0.358 \\
\hline Oxygen requirement & $5(12.8)$ & $4(6.6)$ & $2.10(0.45,10.14)$ & 0.306 \\
\hline Composite neonatal morbidity & $14(35.9)$ & $21(34.4)$ & $1.07(0.42,2.69)$ & 0.880 \\
\hline Placental weight (g) & $355.4 \pm 73.8$ & $354.8 \pm 82.1$ & & 0.972 \\
\hline Birth/placental weight ratio & $6.75 \pm 1.19$ & $6.89 \pm 1.37$ & & 0.594 \\
\hline Umbilical cord diameter $(\mathrm{cm})$ & $1.24 \pm 0.32$ & $1.18 \pm 0.28$ & & 0.309 \\
\hline Placental weight $<5$ th percentile & $17(43.6)$ & $23(37.7)$ & $1.23(0.52,3.14)$ & 0.558 \\
\hline
\end{tabular}

$\mathrm{OR}=$ odds ratio, $\mathrm{CI}=$ confidence interval, and $\mathrm{FGR}=$ fetal growth restriction.

${ }^{*}$ Results expressed in mean $\pm \mathrm{SD}$, number (\%), or median (min-max).

In suspected FGR, the presence of placental pathology could be seen as validating a placental cause for the FGR. If we considered the presence of placental pathology as representing "true" or "pathologic" FGR and the absence of placental pathology representing the constitutionally small normal fetus, our study would indicate that the latter population of fetuses $(31 \%)$ actually represents a minority of suspected FGR delivered at 37 weeks. MCA Doppler had high specificity and positive predictive value for placental disease and theoretically "true" growth restriction; however it had many false negatives and consequently low negative predictive value. Ideally, one would want to exclude the constitutionally small normal fetus, so that this group could be managed with less surveillance and without mandated early term delivery; our study suggests that this separation cannot be accomplished with Doppler; thus all cases with suspected FGR would have to be managed similarly. Having an agreedupon postnatal reference standard as to what constitutes 
TABLE 10: Abnormal versus normal Doppler antenatal comparisons in suspected FGR data for $n=68$ subjects with both umbilical artery and MCA Doppler evaluation.

\begin{tabular}{lccc}
\hline Variable & Abnormal Doppler & Normal Doppler & OR (95\% CI) \\
& $N=41$ & $N=27$ & 0.065 \\
Maternal age & $25.1 .2 \pm 5.8$ & $27.0 \pm 6.4$ & 0.065 \\
Maternal BMI & $29.8 \pm 6.8$ & $27.6 \pm 5.2$ & 0.065 \\
Caucasian & $24(58.5)$ & $22(81.5)$ & $0.32(0.09,1.14)$ \\
Diabetes & $4(9.8)$ & $1(3.7)$ & $2.81(0.27,70.0)$ \\
Hypertension & $3(7.3)$ & 0 & 0.641 \\
Parity $\geq 1$ & $19(46.3)$ & $17(63)$ & $0.51(0.17,1.53)$ \\
Smoking & $15(36.6)$ & $10(37.0)$ & $0.98(0.32,3.03)$ \\
EFW ultrasound (grams) & $2120.3 \pm 198.9$ & $2230.7 \pm 176.1$ & 0.179 \\
EFW $<$ 3rd percentile & $13(31.7)$ & $3(11.1)$ & 0.970 \\
HC/AC $>$ 95th percentile & $15(36.6)$ & $8(29.6)$ & 0.022 \\
Induction of labor & $34(82.9)$ & $20(74.1)$ & $1.37(0.43,4.43)$ \\
Vaginal delivery & $33(71.7)$ & $41(75.9)$ & $1.70(0.45,6.78)$ \\
Cesarean for nonreassuring fetal status & $5(12.9)$ & 0 & 0.079 \\
Vacuum/forceps vaginal delivery & $1(3.6)$ & 0 & $0.553(0.30,2.16)$ \\
\hline
\end{tabular}

$\mathrm{OR}=$ odds ratio, $\mathrm{CI}=$ confidence interval, and FGR $=$ fetal growth restriction.

${ }^{*}$ Results expressed in mean \pm SD or number (\%).

TABLE 11: Abnormal versus normal Doppler newborn comparisons in suspected FGR data for $n=68$ subjects with both umbilical artery and MCA Doppler evaluation.

\begin{tabular}{lccc}
\hline Variable* & Abnormal Doppler & Normal Doppler & OR (95\% CI) \\
\hline Birthweight $(\mathrm{g})$ & $N=41$ & $N=27$ & 0.019 \\
SGA & $2269.0 \pm 253.3$ & $2449.2 \pm 177.0$ & 0.025 \\
Ponderal index $\left(\mathrm{g} / \mathrm{cm}^{3}\right)$ & $14(34.1)$ & $6(27)$ & 0.379 \\
Ponderal index $<10$ th percentile & $2.37 \pm 0.33$ & $2.43 \pm 0.33$ & $0.13 .02,9.83)$ \\
Five-minute Apgar $<7$ & $14(34.1)$ & $6(22.2)$ & $1.82(0.53,6,4)$ \\
Hospital stay (days) & 0 & 0 & 0.291 \\
NICU admission & $3(2-16)$ & $3(2-12)$ & $0.98(0.27,3.71)$ \\
Hyperbilirubinemia phototherapy & $9(22.0)$ & $6(22.2)$ & $1.62(0.39,7.2)$ \\
Hypoglycemia & $9(22.0)$ & $4(14.8)$ & 0.570 \\
Hypothermia & $6(14.6)$ & 0 & 0.979 \\
Oxygen requirement & $5(12.2)$ & $3(11.1)$ & 0.464 \\
Composite neonatal morbidity & $4(9.8)$ & $2(7.4)$ & 0.074 \\
Placental weight g) & $16(39)$ & $5(18.5)$ & $1.35(0.19,11.63)$ \\
Birth/placental weight ratio & $353.0 \pm 74.4$ & $378.4 \pm 88.2$ & $2.82(0.79,10.58)$ \\
Umbilical cord diameter $(\mathrm{cm})$ & $6.60 \pm 1.09$ & $6.70 \pm 1.26$ & 1.000 \\
Placental weight $<$ th percentile & $1.20 \pm 0.35$ & $1.19 \pm 0.21$ & 0.073 \\
\hline
\end{tabular}

$\mathrm{OR}=$ odds ratio, $\mathrm{CI}=$ confidence interval, and $\mathrm{FGR}=$ fetal growth restriction .

${ }^{*}$ Results expressed in mean $\pm \mathrm{SD}$, number $(\%)$, or median (min-max).

"true" or "pathologic" fetal growth restriction may allow antenatal separation of the constitutionally small normal fetus from the truly growth restricted fetus in the future.

\section{Conclusion}

Abnormal Doppler ultrasound was significantly associated with the presence of placental pathology in this group of singleton pregnancies delivered at 37 weeks' gestation for suspected FGR. Of the three Doppler types evaluated, an abnormal MCA Doppler had the strongest association with the presence of placental pathology. This study provides further evidence and support for the use of MCA Doppler in the evaluation of suspected FGR and underscores the limitation of umbilical artery Doppler alone in FGR at later gestational ages. Further investigation and tools for separating the constitutionally small normal fetus from the FGR fetus are needed. 
TABLE 12: Prevalence of placental pathology: abnormal versus normal Doppler in suspected FGR for $n=68$ subjects with umbilical and MCA Doppler evaluations.

\begin{tabular}{|c|c|c|c|c|c|c|}
\hline \multirow{3}{*}{ Doppler type } & \multirow{2}{*}{\multicolumn{2}{|c|}{$\begin{array}{l}\text { Abnormal Doppler } \\
\text { Placental pathology* }\end{array}$}} & \multirow{2}{*}{\multicolumn{2}{|c|}{$\begin{array}{l}\text { Normal Doppler } \\
\text { Placental pathology }\end{array}$}} & \multirow{3}{*}{ OR (95\% CI) } & \multirow{3}{*}{$p$ value } \\
\hline & & & & & & \\
\hline & Yes & No & Yes & No & & \\
\hline Any & $35(85.4)$ & $6(14.6)$ & $13(48.1)$ & $14(51.9)$ & $6.28(1.75,23.5)$ & 0.001 \\
\hline Umbilical & $9(75.0)$ & $3(25.0)$ & $39(69.6)$ & $17(30.4)$ & $1.31(0.27,7.02)$ & 1.000 \\
\hline MCA & $25(96.2)$ & $1(3.8)$ & $23(54.8)$ & $19(45.2)$ & $20.7(2.54,447.1)$ & $<0.001$ \\
\hline Uterine & $15(88.2)$ & $2(11.8)$ & $13(61.9)$ & $8(38.1)$ & $4.62(0.69,38.6)$ & 0.136 \\
\hline
\end{tabular}

$\mathrm{OR}=$ odds ratio, $\mathrm{CI}=$ confidence interval, $\mathrm{MCA}=$ middle cerebral artery, and $\mathrm{FGR}=$ fetal growth restriction

${ }^{*}$ Results expressed in number (\%).

\section{Conflicts of Interest}

The authors declare that they have no conflicts of interest.

\section{References}

[1] American College of Obstetricians and Gynecologists, ACOG Practice Bulletin, Number 134: Intrauterine Growth Restriction, American College of Obstetricians and Gynecologists, Washington, DC, USA, 2013.

[2] S. J. Gordijn, I. M. Beune, B. Thilaganathan et al., "Consensus definition of fetal growth restriction: a Delphi procedure," Ultrasound in Obstetrics \& Gynecology, vol. 48, no. 3, pp. 333339, 2016.

[3] M. S. Anderson and W. W. Hay, "Intrauterine growth restriction and the small-for-gestational-age infant," in Neonatology Pathophysiology and Management of the Newborn, G. B. Avery, M. A. Fletcher, and M. G. MacDonald, Eds., p. 411, Lippincott Williams and Wilkins, Philadelphia, Pa, USA, 5th edition, 1999.

[4] H. C. Miller and K. Hassanein, "Diagnosis of impaired fetal growth in newborn infants," Pediatrics, vol. 48, no. 4, pp. 511522, 1971.

[5] J. A. Bates, J. A. Evans, and G. Mason, "Differentiation of growth retarded from normally grown fetuses and prediction of intrauterine growth retardation using Doppler ultrasound," British Journal of Obstetrics and Gynaecology, vol. 103, no. 7, pp. 670-675, 1996.

[6] B. Clausson, S. Cnattingius, and O. Axelsson, "Outcomes of post-term births: the role of fetal growth restriction and malformations," Obstetrics and Gynecology, vol. 94, no. 5, pp. 758-762, 1999.

[7] D. Getahun, C. V. Ananth, and W. L. Kinzler, "Risk factors for antepartum and intrapartum stillbirth: a population-based study," American Journal of Obstetrics and Gynecology, vol. 196, no. 6, pp. 499-507, 2007.

[8] H. L. Galan, "Timing delivery of the growth-restricted fetus," Seminars in Perinatology, vol. 35, no. 5, pp. 262-269, 2011.

[9] Z. Alfirevic, T. Stampalija, and G. M. L. Gyte, "Fetal and umbilical Doppler ultrasound in high-risk pregnancies," The Cochrane Database of Systematic Reviews, vol. 11, Article ID CD007529, 2013.

[10] C. M. Salafia, J. C. Pezzullo, V. K. Minior, and M. Y. Divon, "Placental pathology of absent and reversed end-diastolic flow in growth- restricted fetuses," Obstetrics and Gynecology, vol. 90, no. 5, pp. 830-836, 1997.

[11] S. Pathak, C. C. Lees, G. Hackett, F. Jessop, and N. J. Sebire, "Frequency and clinical significance of placental histological lesions in an unselected population at or near term," Virchows Archiv, vol. 459, no. 6, pp. 565-572, 2011.

[12] M. Parra-Saavedra, F. Crovetto, S. Triunfo et al., "Association of Doppler parameters with placental signs of underperfusion in late-onset small-for-gestational-age pregnancies," Ultrasound in Obstetrics and Gynecology, vol. 44, no. 3, pp. 330-337, 2014.

[13] American College of Obstetricians and Gynecologists, ACOG Practice Bulletin Number 101: Ultrasonography in Pregnancy, American College of Obstetricians and Gynecologists, Washington, DC, USA, 2009.

[14] F. P. Hadlock, R. B. Harrist, R. S. Sharman, R. L. Deter, and S. K. Park, "Estimation of fetal weight with the use of head, body, and femur measurements-a prospective study," American Journal of Obstetrics and Gynecology, vol. 151, no. 3, pp. 333-337, 1985.

[15] F. P. Hadlock, R. B. Harrist, and J. Martinez-Poyer, "In utero analysis of fetal growth: a sonographic weight standard," Radiology, vol. 181, no. 1, pp. 129-133, 1991.

[16] G. Acharya, T. Wilsgaard, G. K. R. Berntsen, J. M. Maltau, and T. Kiserud, "Reference ranges for serial measurements of umbilical artery Doppler indices in the second half of pregnancy," American Journal of Obstetrics and Gynecology, vol. 192, no. 3, pp. 937-944, 2005.

[17] C. Ebbing, S. Rasmussen, and T. Kiserud, "Middle cerebral artery blood flow velocities and pulsatility index and the cerebroplacental pulsatility ratio: longitudinal reference ranges and terms for serial measurements," Ultrasound in Obstetrics and Gynecology, vol. 30, no. 3, pp. 287-296, 2007.

[18] O. Gómez, F. Figueras, S. Fernández et al., "Reference ranges for uterine artery mean pulsatility index at 11-41 weeks of gestation," Ultrasound in Obstetrics and Gynecology, vol. 32, no. 2, pp. 128$132,2008$.

[19] C. Langston, C. Kaplan, T. Macpherson et al., "Practice guideline task force of the College of American Pathologists," Archives of Pathology \& Laboratory Medicine, vol. 121, no. 5, pp. 449-476, 1997.

[20] F. T. Kraus, R. W. Redline, D. J. Gesell, D. M. Nelson, and J. M. Dicke, "Appendix: placental weights and measures," in Atlas of Non-Tumor Pathology: Placental Pathology, pp. 311-317, American Registry of Pathology, 2004.

[21] G. Altshuler, "Chorangiosis. An important placental sign of neonatal morbidity and mortality," Archives of Pathology and Laboratory Medicine, vol. 108, no. 1, pp. 71-74, 1984.

[22] R. W. Redline, T. Boyd, V. Campbell et al., "Maternal vascular underperfusion: nosology and reproducibility of placental reaction patterns," Paediatric \& Developmental Pathology, vol. 7, no. 3, pp. 237-249, 2004. 
[23] R. W. Redline, I. Ariel, R. N. Baergen et al., "Fetal vascular obstructive lesions: nosology and reproducibility of placental reaction patterns," Pediatric and Developmental Pathology, vol. 7, no. 5, pp. 443-452, 2004.

[24] J. M. D. Thompson, L. M. Irgens, R. Skjaerven, and S. Rasmussen, "Placenta weight percentile curves for singleton deliveries," BJOG, vol. 114, no. 6, pp. 715-720, 2007.

[25] J. M. Dicke, P. Huettner, S. Yan, A. Odibo, and F. T. Kraus, "Umbilical artery Doppler indices in small for gestational age fetuses: correlation with adverse outcomes and placental abnormalities," Journal of Ultrasound in Medicine, vol. 28, no. 12, pp. 1603-1610, 2009.

[26] S. Savchev, F. Figueras, M. Sanz-Cortes et al., "Evaluation of an optimal gestational age cut-off for the definition of early- and late-onset fetal growth restriction," Fetal Diagnosis and Therapy, vol. 36, no. 2, pp. 99-105, 2014.

[27] M. Parra-Saavedra, F. Crovetto, S. Triunfo et al., "Placental findings in late-onset SGA births without Doppler signs of placental insufficiency," Placenta, vol. 34, no. 12, pp. 1136-1141, 2013.

[28] L. M. E. McCowan, J. E. Harding, and A. W. Stewart, "Umbilical artery Doppler studies in small for gestational age babies reflect disease severity," British Journal of Obstetrics and Gynaecology, vol. 107, no. 7, pp. 916-925, 2000.

[29] T. M. Macdonald, E. A. McCarthy, and S. P. Walker, "Shining light in dark corners: diagnosis and management of late-onset fetal growth restriction," Australian and New Zealand Journal of Obstetrics and Gynaecology, vol. 55, no. 1, pp. 3-10, 2015.

[30] F. Figueras, R. Cruz-Martinez, M. Sanz-Cortes et al., "Neurobehavioral outcomes in preterm, growth-restricted infants with and without prenatal advanced signs of brain-sparing," Ultrasound in Obstetrics and Gynecology, vol. 38, no. 3, pp. 288294, 2011.

[31] K. E. Boers, L. Van Wyk, J. A. M. Van Der Post et al., "Neonatal morbidity after induction vs expectant monitoring in intrauterine growth restriction at term: a subanalysis of the DIGITAT RCT," American Journal of Obstetrics and Gynecology, vol. 206, no. 4, pp. 344.el-344.e7, 2012. 


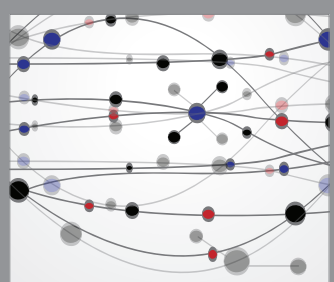

The Scientific World Journal
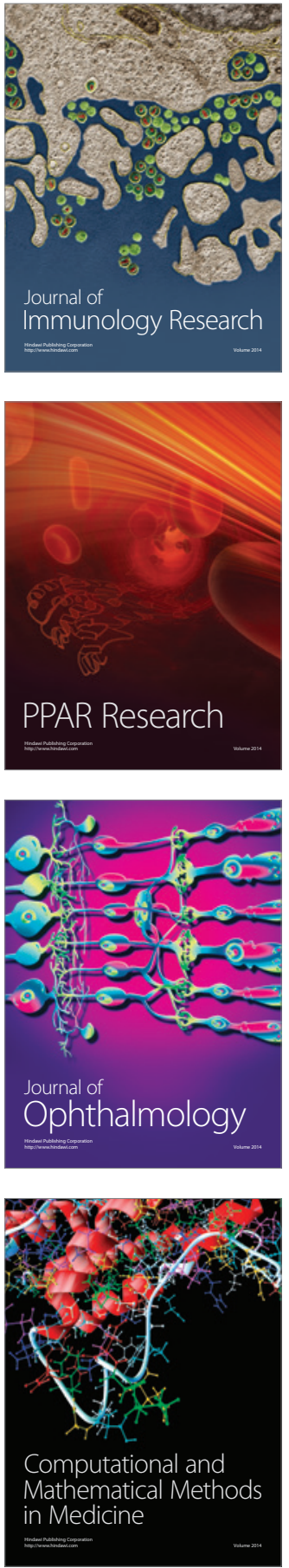

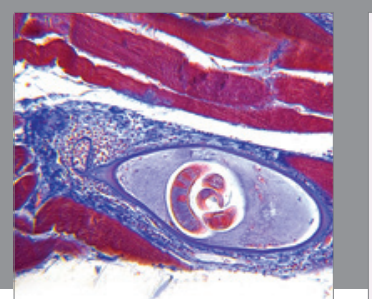

Gastroenterology Research and Practice
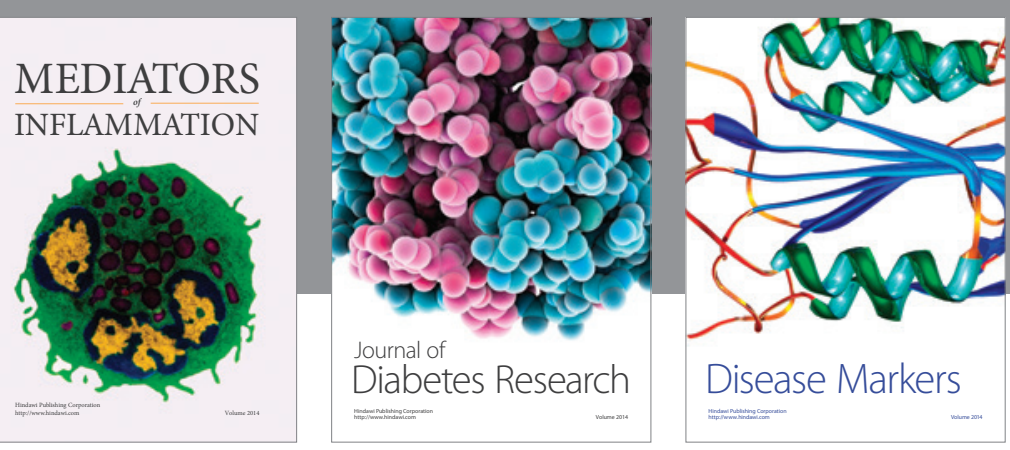

Disease Markers

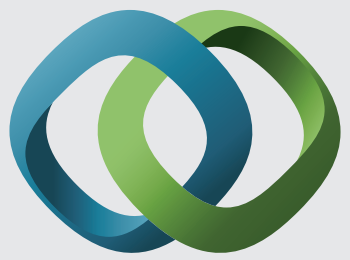

\section{Hindawi}

Submit your manuscripts at

https://www.hindawi.com
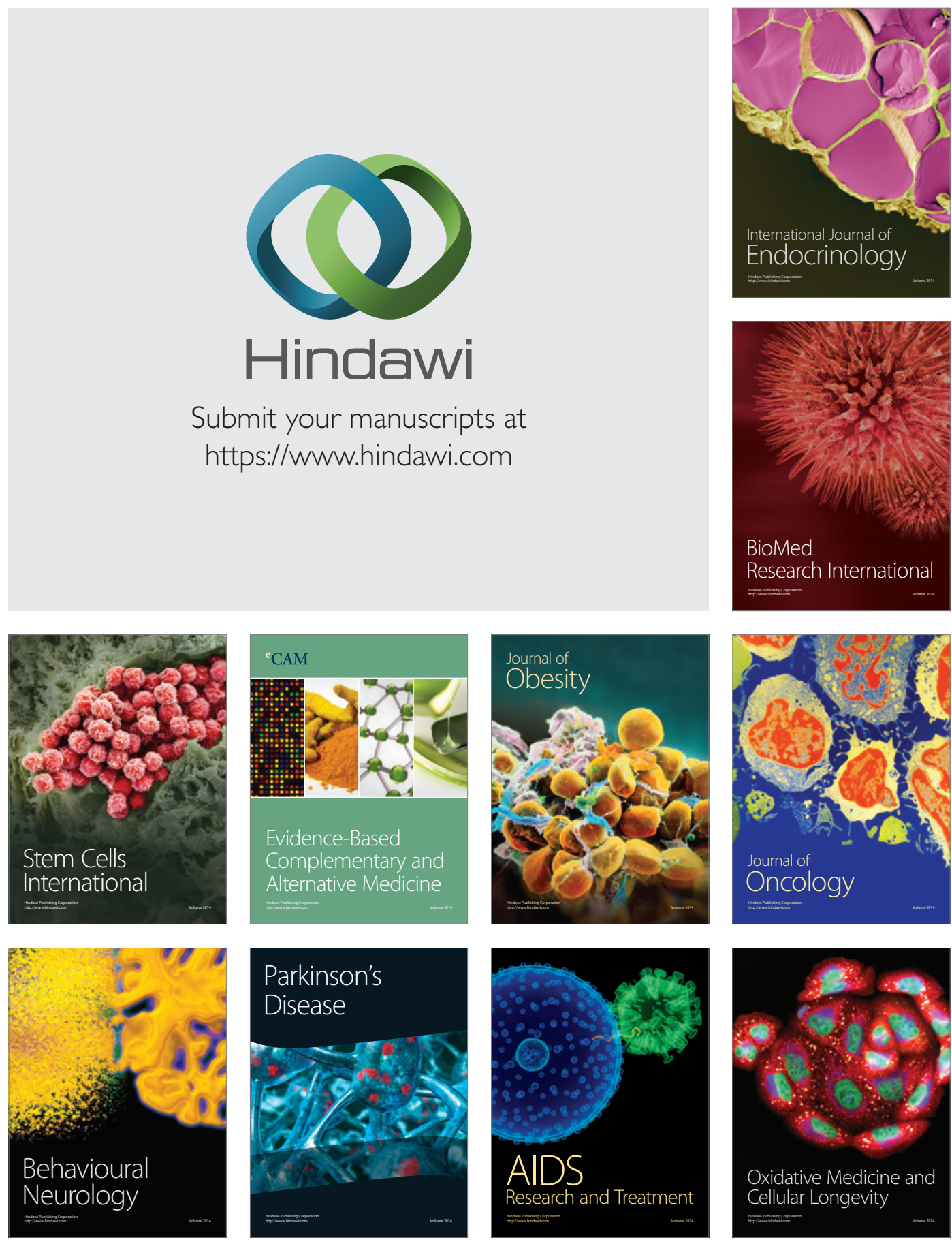